\title{
SPATIALLY LOCAL MINIMIZERS OF THE LANDAU-GINZBURG FUNCTIONAL*
}

BY

\author{
E. B. NAUMAN AND N. P. BALSARA
}

Rensselaer Polytechnic Institute, Troy, New York

1. Introduction. The free energy of a spatially inhomogeneous mixture can depend on concentration gradients in addition to the normal dependence on concentration. The lowest-order, nontrivial approximation of this dependence is given by the so-called Landau-Ginzburg functional. For binary mixtures and a single spatial dimension, the functional is

$$
G_{\text {total }}=\int_{-L / 2}^{L / 2}\left[g+\frac{\kappa}{2}\left(\frac{d a}{d z}\right)^{2}\right] d z .
$$

Here, $a=a(z)$ is the mole fraction of component $A$, and $g=g(a)$ is the Gibbs free energy for a well-mixed solution of concentration $a, 0 \leq a \leq 1$. The gradient energy parameter, $\kappa \geq 0$, is assumed to be constant with respect to $a$ and $z$.

Equation (1.1) was apparently first derived by van der Waals; see Rowlinson [1]; but its current popularity is attributable to Cahn and Hilliard [2] who used the functional to analyze phase transition mechanisms. Interest in (1.1) is focused on a set of Gibbs functions, $g(a)$, which we regard as defined and continuous with continuous derivatives over $0 \leq a \leq 1$. The Gibbs functions of physical interest have local maxima at $a=0$ and $a=1$, two interior minima, and one interior maximum. The maximum lies inside the spinodal which is the region bounded by the two roots of $g^{\prime \prime}=0$. More precise restrictions on $g$ have been given by Carr, Gurtin, and Slemrod [3]. This class of Gibbs functions corresponds to physical mixtures which spontaneously bifurcate into two phases, at least when the average concentration, $\bar{a}$, lies inside the spinodal and when $\kappa$ is sufficiently small.

A mathematical bifurcation arises naturally from the minimization of Eq. (1.1) subject to the material balance constraint that

$$
\int_{-L / 2}^{L / 2} a d z=\bar{a} .
$$

This minimization of (1.1) subject to (1.2) has been considered by Novick-Cohen and Segel [4], Carr, Gurtin, and Slemrod [3], [5], and Nauman and Balsara [6]. For $\kappa>0$ the global minimizing functions are known to be monotone and to satisfy the

\footnotetext{
${ }^{*}$ Received July 14, 1987.
} 
natural boundary conditions:

$$
\frac{d a}{d z}=0 \quad \text { at } z= \pm L / 2 .
$$

Thus the minimizing functions can be regarded as half-waves of a periodic function. Full period and multiple period solutions do not yield global minima for $G_{\text {total }}$.

The global minima correspond to true thermodynamic equilibria. The present paper is concerned with spontaneous processes which lower $G_{\text {total }}$ but do not necessarily achieve true equilibrium. It has long been a tenet of thermodynamics that such states can exist. They correspond to local minima in $G_{\text {total }}$ and are locally stable. The concentration profiles have ceased to evolve with time, but true equilibrium has not been attained. This belief is confronted by an argument due to Carr, Gurtin, and Slemrod [3] that nonmonotonic $a(z)$ are unstable and cannot be even local minimizers of $G_{\text {total }}$. We shall see that their result, although mathematically correct, uses a nonphysical definition for local.

Periodic, quasi-equilibrium minimizers of $(1.1)$ are generated by numerical solutions [4,7] of the Cahn-Hilliard diffusion equation [8]. Our solutions, using finite difference approximations, exhibited dominant periods greater than the minimum size for growth as predicted by linear stability theory:

$$
\alpha_{c}=\sqrt{\frac{-g^{\prime \prime}(\bar{a})}{\kappa}} .
$$

Short-time solutions are sinusoidal, but the waveform flattens as the maximum and minimum concentrations approach the binodal points. Our long-time solutions to the Cahn-Hilliard equation were periodic, or nearly so, and appeared to be stationary. They were clearly suboptimal due to the multiple interfaces. Numerical solutions using finite element methods have been reported by Elliott and French [7]. These too give apparently stable, suboptimal concentration profiles. Physical results from spinodal decomposition also give suboptimal profiles with multiple interfaces that are stable over extended periods. All of these examples show a form of metastability which is not encompassed by the theory of Carr, Gurtin, and Slemrod [3]. Their theory shows that periodic solutions are unstable to small concentration disturbances if no limits are imposed on the spatial extent of the distrubance. The present paper considers concentration disturbances which are small in both magnitude and spatial extent.

2. Spatially local minimizers. By a spatially local minimizer of $G_{\text {total }}$ we mean a concentration profile, $a(z)$, such that $G_{\text {total }}$ increases when $a(z)$ is subjected to small and localized disturbances. Suppose a concentration fluctuation, $\xi \eta(z)$, is added to $a(z)$. Then $a(z)$ is a spatially local minimizer provided

$$
G_{\text {total }}(a(z)) \leq G_{\text {total }}(a(z)+\xi \eta(z))
$$

for all sufficiently small $\xi>0$. We require that $\eta(z)$ satisfy the material balance constraint that

$$
\int_{-L / 2}^{L / 2} \eta(z) d z=0
$$


To have a physical interpretation as a mole fraction, $0 \leq a+\eta \leq 1$. Furthermore, the distrubance must be localized so that $\eta(z)$ vanishes outside some small but nonzero interval:

$$
\eta(z)=0 \quad \text { if } z>z^{\prime}+\delta \text { or } z<z^{\prime}-\delta
$$

for some $-L / 2<z^{\prime}<L / 2$ and $\delta>0$. Equations (2.1) and (2.2) are equivalent to those used by Carr, Gurtin, and Slemrod [3] in their definition of a local minimizer. Our definition of a spatially local minimizer imposes Eq. (2.3) as an additional restriction on $\eta(z)$. The $\eta(z)$ so restricted are said to be local to $z^{\prime}$.

Sufficient conditions for $a(z)$ to be a spatially local minimizer are that $a(z)$ satisfy the Euler-Lagrange equation and that

$$
\left.\frac{d^{2} G_{\text {total }}}{d \xi^{2}}\right|_{\xi=0}=\int_{z^{\prime}-\delta}^{z^{\prime}+\delta}\left[g^{\prime \prime}(a) \eta^{2}+\kappa\left(\frac{d \eta}{d z}\right)^{2}\right] d z \geq 0 .
$$

We now construct a nonmonotonic function which will be a spatially local minimizer of $G_{\text {total }}$. Consider the half-interval, $-L / 2 \leq z \leq 0$, and suppose that $a(z)$ is the halfwave, global minimizer over the half-interval. For the second half-interval, $0 \leq$ $z \leq+L / 2$, we use the reversal of $a(z)$. This gives a full-wave function which is symmetric about the origin. Consider some small displacements which are local to $z^{\prime}$. For $-L / 2 \leq z^{\prime}<0$ or $0<z^{\prime} \leq L / 2$, Equation (2.1) must be satisfied since $a(z)$ and $a(-z)$ are global minimizers over the half-intervals. Thus we need consider only those concentration fluctuations which are local to $z^{\prime}=0$. Note that $a(0)$ will be a maximum or minimum; and except for very large $\kappa, a(0)$ will lie outside the spinodal so that $g^{\prime \prime}(a(0))>0$. We can thus choose $\delta>0$ such that $a(z)$ lies outside the spinodal for all $z$ in the interval $-\delta \leq z \leq \delta$. This means that (2.4) is satisfied since every term in the integral is positive. Hence the full-wave solution is a spatially local minimizer of $G_{\text {total }}$ provided the extreme values for $a(z)$ lie outside the spinodal.

To treat the case of $a(0)$ being inside the spinodal, it is first useful to consider flat profiles, $a(z)=\bar{a}$, which lie within the spinodal. Can these be spatially local minimizers? For a given $\eta(z)$, it is clear that $\kappa$ can always be chosen large enough so that $(2.4)$ is satisfied. However, we must consider all possible $\eta(z)$ that are local to $z^{\prime}$. This introduces a problem in the calculus of variations: Find the $\eta(z)$ which minimizes the integral in (2.4) and then see if (2.4) remains true for this minimizing function. In what follows, it is convenient to set $z^{\prime}=0$ so that the integral in (2.4) becomes

$$
I=\int_{-\delta}^{\delta}\left[g^{\prime \prime}(a) \eta^{2}+\kappa\left(\frac{d \eta}{d z}\right)^{2}\right] d z
$$

subject to the constraint that

$$
\int_{-\delta}^{\delta} \eta d z=0
$$

The Euler-Lagrange equation corresponding to (2.5) and (2.6) is

$$
\frac{d^{2} \eta}{d z^{2}}+\left(\frac{-g^{\prime \prime}(a)}{\kappa}\right) \eta+\rho=0
$$


where $\rho$ is a constant. The case of $a(z)=\bar{a}$ gives an equation with constant coefficients:

$$
\frac{d^{2} \eta}{d z^{2}}+\alpha_{c}^{2} \eta+\rho=0
$$

where $\alpha_{c}$ is given by (1.4). A solution which satisfies (2.8), (2.6), and the natural boundary conditions is

$$
\eta=\sin \alpha_{c} z, \quad 0 \leq z \leq \pi / \alpha_{c}
$$

so that $\delta=\pi / \alpha_{c}$ is now prescribed. It can be shown that $(2.8)$ is indeed the minimizing solution. Inserting it into (2.5) gives $I=0$ so that the flat profile is a spatially local minimizer.

Now consider the full-wave function treated earlier but suppose $a(0)$ lies inside the spinodal. Observe that $g(a(0))$ is a relative maximum so that $-g(a(0)) \leq-g(a( \pm \delta))$. Pick $\delta>0$ such that

$$
\delta \ll \pi / \alpha_{c}=\sqrt{\frac{\pi^{2} \kappa}{-g^{\prime \prime}(a(0))}} .
$$

This will ensure that

$$
\delta<\sqrt{\frac{\pi^{2} \kappa}{-g^{\prime \prime}(a \pm \delta)}} .
$$

With this $\delta, I$ will be nonnegative even using the lowest value of $g^{\prime \prime}$ over $-\delta \leq z \leq \delta$. Thus the full-wave solution is a spatially local minimizer for $G_{\text {total }}$ for any $a(0)$.

3. Discussion. We have shown that binary mixtures, of the type that form two equilibrium phases when $\kappa$ is small, admit spatially local minimizing solutions in a variety of nonequilibrium situations. The flat composition profiles, $a(z)=\bar{a}$, are spatially local minimizers throughout the composition range. If $\bar{a}$ lies outside the spinodal, $g^{\prime \prime}(\bar{a}) \geq 0$; and Eq. (2.4) is satisfied for any $\delta$. This profile is also a local minimizer in the sense of Carr, Gurtin, and Slemrod [3]. The flat profile with $\bar{a}$ outside the spinodal will withstand concentration fluctuations unlimited in extent provided they remain small in magnitude. Large scale concentration fluctions, as encountered in nucleation and growth, will destabilize the system.

When $a(z)=\bar{a}$ but $\bar{a}$ lies inside the spinodal, then $g^{\prime \prime}(\bar{a})<0$. These flat concentration profiles are not local minimizers in the sense of Carr, Gurtin, and Slemrod [3] but are spatially local minimizers in the sense used here provided

$$
\delta<\pi / \alpha_{c}=\sqrt{\frac{\pi^{2} \kappa}{-g^{\prime \prime}(\bar{a})}} .
$$

This distance is one-half the critical wavelength predicted by Cahn [1968] in his linearized theory of spinodal decomposition. It is also one-half the minimum size for two-phase bifurcation when $\bar{a}$ is deep within the spinodal as predicted by Nauman and Balsara [6]. The same limiting parameter has now been found through three quite different approaches.

Carr, Gurtin, and Slemrod found that nonmonotonic $a(z)$ could not be even local minimizers. What this means is that nonmonotonic structures cannot withstand 
concentration fluctuations that are small in magnitude but large in spatial extent. Their proof utilized a concentration fluctuation, $\eta(z)$, which transferred material across the maximum (or minimum) in $a(z)$. They used an $\eta(z)$ which is nonzero over distances larger than $\pm \delta$ where $\delta$ is given by (2.12).

We, however, have shown that periodic $a(z)$ can be spatially local minimizers of $G_{\text {total }}$. Such structures would be stable to concentration distrubances which are confined in both magnitude and spatial extent. This finding agrees with experimental observations on spinodally decomposed systems and with long-time, numerical solutions of the Cahn-Hilliard equation. However, there is not a one-to-one relationship between spatially local minimizers and (local) stability. The flat profiles with $\bar{a}$ inside the spinodal are spatially local minimizers provided $\delta$ satisfies (3.1). Numerical simulations show that disturbances which initially satisfy (3.1) decline in magnitude but grow in spatial extent. Eventually, the spatial extent of the disturbance violates (3.1) and the system becomes unstable. It happens that numerical solutions then approach one of the periodic, spatially local minimizers considered above. These, in turn, are stable to further small, spatially confined disturbances. The precise relationship between spatially local minimizers and local stability thus remains to be elucidated.

\section{REFERENCES}

[1] J. S. Rowlinson, Translation of J. D. van der Waals' "The Thermodynamic Theory of Capillarity Under the Hypothesis of a Continuous Variation of Density", J. Stat. Phys. 20, 197-244 (1979)

[2] J. W. Cahn and J. E. Hilliard, Free Energy of a Nonuniform System, I. Interfacial Free Energy, J. Chem. Physics 28, 258-267 (1958)

[3] J. Carr, M. E. Gurtin, and M. Slemrod, Standard Phase Transition on a Finite Interval, Arch. Rat. Mech. Anal. 86, 317-351 (1984)

[4] A. Novick-Cohen and L. A. Segel, Nonlinear Aspects of the Cahn-Hilliard Equation, Physica 10D, 277-298 (1984)

[5] J. Carr, M. E. Gurtin, and M. Slemrod, One-Dimensional Structured Phase Transformations Under Prescribed Loads, J. Elasticity 15, 133-142 (1985)

[6] E. B. Nauman and N. Balsara, Phase Equilibria and the Landau-Ginzburg Functional, to appear (1988)

[7] C. N. Elliott and D. A. French, Numerical Studies of the Cahn-Hilliard Equation for Phase Separation, to appear (1988)

[8] J. W. Cahn, Spinodal Decomposition, Trans. Met. Soc. AIME, 242, 166-180 (1968) 